\title{
THE WESTERN CHRISTMAS MEETING OF THE SOCIETY AT CHICAGO
}

The twenty-sixth Western meeting of the Society was held at the University of Chicago on Friday, December 31, 1926. It throws an interesting light upon the growth of the Society that, in spite of the strong attractive power of the meeting in Philadelphia, the attendance at this meeting ran up to about 75, among whom were the following 54 members of the Society, a number which would have been counted large for a meeting ten or twelve years ago:

Frank Ayres, R. W. Babcock, Barnett, Ethelwynn R. Beckwith, Brenke, Clack, H. T. Davis, Dickson, Dresden, Edington, H. P. Evans, Feltges, Frink, Garver, Georges, G. H. Graves, L. M. Graves, Griffiths, V. G. Grove, Harshbarger, Hickson, Hildebrandt, Hillard, Hodge, Rosa L. Jackson, LaPaz, Logsdon, W. D. MacMillan, March, William Marshall, Marquis, Mirick, E. H. Moore, E. J. Moulton, A. L. Nelson, Newson, Nyswander, Pettit, Rainich, Roos, Roth, Schottenfels, Sheffer, Shohat, Simmons, Skinner, J. H. Taylor, E. L. Thompson, J. S. Turner, Van Vleck, Wait, G. T. Whyburn, John Williamson, F. E. Wood.

It was agreed to hold the spring meeting in Chicago on April 15 and 16,1927. At that meeting Professor Chittenden of the University of Iowa will give the symposium lecture on Some phases of general topology.

The following thirty papers were read at the two sessions on Friday. The first session was presided over by Professor Dickson, relieved by Professor Hildebrandt; the afternoon session was presided over by Professor Van Vleck. Mr. Sheffer, Dr. Trjitzinsky, and Professor Uspensky were introduced to the Society by Professor Dresden. The fourth paper of G. T. Whyburn, and the papers by Trjitzinsky, W. M. Whyburn, Campbell, Coble, Dodd, Roos, Miller, and Manning were read by title.

1. Mr. G. T. Whyburn: Cyclicly connected continuous curves.

In this paper a new type of continuous curve is studied. A continuous curve $M$ is said to be cyclicly connected if and only if every two points of 
$M$ lie together on some simple closed curve which is contained in $M$. A cyclicly connected continuous curve $C$ which is a subset of a continuous curve $M$ is a maximal cyclic curve of $M$ provided $C$ is not a proper subset of any other cyclicly connected continuous curve which is a subset of $M$. The most important theorems proved are as follows: (1) A continuous curve $M$ is cyclicly connected if and only if $M$ has no cut point. (2) If $M$ is a continuous curve and $J$ is any simple closed curve of $M$, then $M$ contains a maximal cyclic curve which contains $J$. (3) If $N$ denotes the point set obtained by adding together all the simple closed curves contained in a continuous curve $M$, then $N$ is the sum of a countable number of maximal cyclic curves of $M$. (4) If $H$ is a maximal connected subset of $N$, then $\bar{H}$ ( $H$ plus its limit points) is a two-way continuous curve.

\section{Mr. G. T. Whyburn: Some properties of continuous} curves.

This paper will appear in full in an early issue of this Bulletin.

\section{Mr. G. T. Whyburn: Concerning the structure of a continuous curve.}

The author shows that if the cyclic elements of a continuous curve $M$ are defined as follows: $E$ is a cyclic element of $M$ provided either $E$ is a maximal cyclic curve of $M$ or $E$ is a point of $M$ not belonging to any maximal cyclic curve of $M$, then every continuous curve is acyclic with respect to its cyclic elements. The following familiar properties of acyclic continuous curves are established for any continuous curve $M$ which is regarded as being composed of its cyclic elements: (1) every collection of whole elements of $M$ whose sum is a connected point set is arcwise connected in the ordinary sense and is also cyclic chainwise connected; and every such collection whose sum is a continuum is a continuous curve; (2) an element of $M$ is an end element if and only if it is a non-cut element; (3) if $C$ is any simple cyclic chain of elements of $M$, there exists in $M$ a maximal simple cyclic chain which contains $C$.

\section{Mr. G. T. Whyburn: On continuous curves.}

In this paper it is shown that if the continuous curve $N$ is a proper subset of the continuous curve $M$, if $R$ is a maximal connected subset of $M-N$, and $B$ is the boundary of $R$ with respect to $M$, then (1) $R+B$ is a continuous curve, and (2) every maximal connected subset of $B$ is a continuous curve and at most a finite number of these curves are of diameter greater than any preassigned positive number. It is further shown that if the boundary $B$ (with respect to $M$ ) of any $S$-domain $R$ of a continuous curve $M$ either (a) is connected im kleinen, or (b) has no continuum of condensation, then $R+B$ is a continuous curve. An example is given to show that this theorem does not remain true if $B$, instead of satisfying either (a) or (b), merely satisfies the conditions of (2) above. 


\section{Professor G. Y. Rainich: On the definition of differential.}

If the usual definition of differential of a function of several variables or of a vector function is adopted, the existence of the differential does not imply that it is a linear function of the differentials of the independent variables, and that it is a first total differential in the sense of Thomae and Stolz. In this paper another definition of differential as a limit of an expression is proposed and it is proved that if the differential so defined exists, it is a linear function, and that if the approach to the limit is uniform, it is a first total differential (Stolz).

\section{Mr. I. M. Sheffer: On entire function interpolation.}

With every entire function $f(z)$ we associate a function $P(q), q>0$, determined by the absolute values of the Taylor coefficients of $f(z)$. Some simple properties of $P(q)$ are proved, and it is shown that if $a_{n}(n=0,1, \cdots)$ is a given set of numbers, there exist infinitely many entire functions $E(z)$ such that $E(n)=a_{n}$. This theorem, usually proved by means of a theorem due to Mittag-Leffler, follows here from the properties of $P(q)$ in a very elementary manner. It is shown by induction that the first $k$ derivatives of $E(z)$ ( $k$ arbitrary) may also be assigned at $z=0,1, \cdots$. The results are applied to give a new proof of a theorem due to Borel on the singularities of analytic functions whose Taylor coefficients satisfy certain conditions. This leads to an interesting set of polynomials of which some important properties are stated.

7. Dr. W. J. Trjitzinsky: On integral transcendental functions.

It is proved that there exists at least a set of entire functions $F(z)$, such that the expansion $F(z)=a_{1} F\left(\xi_{1} z\right)+a_{2} F\left(\xi_{2} z\right)+\cdots+a_{n} F\left(\xi_{n} z\right)+\cdots$ holds under certain restrictions, $\xi_{i}$ being constant; explicit expressions for the coefficients $a_{i}$ are found as infinite products. Absolute and uniform convergence of the expansion is established and also the equality of the expansion and the function. The expansion is obtained as the limit of a similar terminating expansion in polynomials. The validity of the process is established by the representation of double limits as the sums of terms of an absolutely convergent double array.

\section{Professor W. M. Whyburn: On differential equations} and their related algebraic systems.

The method of successive approximations is used to obtain the solution of a system of $n$ difference equations of the first order. It is proved that this solution tends in the limit to the solution (obtained by the same method) of a system of differential equations whose coefficients are Lebesgue summable functions. The coefficients of the difference system are defined as sequences of horizontal functions having as their limit functions almost everywhere the summable coefficients of the differential system. The above result enables the author to complete the carrying over of 
results previously obtained for difference systems to their related differential systems. See a paper presented to the American Mathematical Society at the summer meeting, September 8-9, 1926.

\section{Professor J. A. Nyswander: A direct method of solving systems of linear differential equations of the Fuchsian type.}

The only exhaustive treatment to be found in the literature of a general system of $n$ linear differential equations of the first order in $n$ dependent variables and one independent variable, the singular points of whose coefficients are poles of the first order, viz., that given by J. Horn about 1890, is based on the theory of transformations and elementary divisors. The present paper gives a new theory which arrives at the general solution, in each of the various cases, by a direct determination of the coefficients of the power series that enter into the solutions. Early in the theory arises the so-called characteristic determinant-equation $D(\lambda)=0$ whose $n$ roots $\lambda_{1}, \lambda_{2}, \cdots, \lambda_{n}$ occur as powers of the independent variables in each of the $n$ solutions respectively. The coefficients that enter into the solutions are obtained, in the more involved cases, from identities in $\lambda$; these identities are built up directly from the determinant $D(\lambda)$.

\section{Professor J. H. Taylor: Concerning an application of} tensor analysis to the first variation of an integral.

J. L. Synge, Proceedings of the London Mathematical Society, (2), vol. 25 (1925), has applied the absolute calculus to the development of the first and second variations of the length integral of a Riemannian space. In the present paper the first variation of an integral in parametric form for the "regular" problem of the calculus of variations is treated in an analogous fashion. The paper is an application of existing theory, as the extensions of the Ricci tensor analysis which are required have been previously developed. (J. H. Taylor, A generalization of Levi-Civita's parallelism and the Frenet formulas, Transactions of this Society, vol. 27 (1925), pp. 246-264.)

\section{Professor V. G. Grove: Transformations of nets.}

In this paper the notion of conjugate nets in the relation of a transformation $F$ (Eisenhart, Jonas) is generalized to any net. Two nets are said to be $C$ transforms, or in relation $C$, if the points of the curves of the nets are in one to one correspondence and the developables of the congruence of lines joining corresponding points cut the sustaining surfaces in the curves of the nets. Thus two conjugate nets in the relation of a transformation $F$ are $C$ transforms. With suitable restrictions on the nets, many properties of conjugate nets in relation $F$ are enjoyed by non-conjugate nets. A generalization of transformation $K$ (Koenig) is made, and, again with suitable restrictions, properties of conjugate nets in the relation of a transformation $K$ are enjoyed by non-conjugate nets. 


\section{Professor A. D. Campbell: Nets of conics in the Galois} fields of order $2^{n}$.

In this paper the nets of conics $\lambda C_{1}+\mu C_{2}+\nu C_{3}=0$ in these finite domains are classified into 32 classes; typical nets are derived for each class. The results of previous papers on pencils of conics and on plane cubic curves in these Galois fields are made use of. In these fields $(a x+b y+c z)^{2}=a^{2} x^{2}+$ $b^{2} y^{2}+c^{2} z^{2}$, the discriminant of the general conic is $f g h+a f^{2}+b g^{2}+c h^{2}$, every point is its own harmonic conjugate with respect to any pair of collinear points. Some cubics in $\lambda, \mu, \nu$ have no associated nets of conics; some nets exist only in the Galois field of order 2, others only when $n>1$, still others only when $2^{n}=3 k+1$; some nets have no degenerate conics in them. When the net has a non-degenerate cubic in $\lambda, \mu, \nu$ this cubic is first reduced to a normal form and then the net is reduced. The nets with degenerate cubics (hence with degenerate pencils of conics in them) are first divided into sets according to the nature of the degenerate pencils they contain.

\section{Professor A. B. Coble: Modular manifolds determined} by the binary $(2 p+2)-i c$.

The cross-ratio group of E. H. Moore is for the binary $(2 p+2)-i c$ a Cremona group of order $(2 p+2)$ ! in a linear space $S_{2 p-1}$. This group has two important invariant linear systems which map the space $S_{2 p-1}$ upon two manifolds of dimension $2 p-1$ in the same higher space. The object of the present paper is to develop some of the geometric properties of these two manifolds and to exhibit their relation to each other.

\section{Professor G. Y. Rainich: Periodic electromagnetic} fields with non-periodic stresses.

Together with an electromagnetic field certain second degree quantities are considered, viz., the stresses, the Poynting vector, and the energy. Given a set of quantities the conditions are established under which they may be considered as second degree quantities of an electromagnetic field, and the question how far the field is determined by these quantities is solved. An example is given in which the field of the second degree quantities is not periodic but determines a periodic electromagnetic field. The possibility of interpreting such fields as radiation is discussed.

15. Professor H. W. March: The Heaviside operational calculus.

This paper will appear in full in an early issue of this Bulletin.

16. Professor W. E. Edington: The relation $s_{2}{ }^{-1} s_{1}{ }^{\epsilon} s_{2}{ }^{\delta} s_{2}=$ $s_{1}^{-1} s_{1}{ }^{\epsilon} s_{2}{ }^{\delta} s_{1}$ in the definition of non-abelian groups of finite order. 
Given $s_{1}^{\alpha}=s_{2}^{\beta}=1, \quad s_{1} s_{2} \neq s_{2} s_{1}, \quad s_{2}^{-1} s_{1}{ }^{\epsilon} s_{2}{ }^{\delta} s_{2}=s_{1}{ }^{-1} s_{1}{ }^{\epsilon} s_{2}{ }^{\delta} s_{1}, \quad \epsilon<\alpha$, $\delta<\beta$ certain general relations exist among some of the operators of the group generated by $s_{1}$ and $s_{2}$ which suggest certain values different from 0 for $\epsilon$ and $\delta$ so as to impose conditions on $\alpha$ and $\beta$ sufficient in some cases to determine infinite systems of non-abelian groups of finite order such as the dihedral and dicyclic systems and several other systems.

\section{Professor J. V. Uspensky: On the development of arbitrary functions in series of Hermite's and Laguerre's polynomials.}

The question concerning the development of arbitrary functions in series of Hermite's and Laguerre's polynomials has recently attracted the attention of several authors. Their results, however, are far less general than those published by the author ten years ago in the Proceedings of the Russian Academy of Sciences. As this paper, published in Russia during the World War did not attract the attention of mathematicians, the author reproduces its contents in a new and simplified form.

18. Professor J. V. Uspensky: On Jacobi's arithmetical theorems contained in his paper: Ueber diejenigen unendlichen Reihen deren Exponenten zugleich in zwei verschiedenen quadratischen Formen enthalten sind.

The author shows how the arithmetical theorems contained in Jacobi's paper mentioned above can be obtained by means of purely arithmetical considerations based upon the study of a certain kind of partitions of numbers.

19. Professor H. T. Davis: A solution of Bourlet's generatrix equation.

C. Bourlet has shown that, in general, the solution of a non-homogeneous linear functional equation $F(x, z) \rightarrow u=f(x)$, where $z^{n}$ is the symbol of the $n$th derivative, can be reduced to that of finding a function $G(x, z)$ which satisfies the generatrix equation $[G \cdot F]=1$. This paper deals with the solution of the equation when $F(x, z)$ is of the form $1-\lambda g(x, z)$ and determines conditions for the existence of the solution regarded as a function of $\lambda$.

20. Professor J. A. Shohat: On the asymptotic expressions of Tchebycheff polynomials and their derivatives.

Consider Tchebycheff polynomials $\phi_{n}(p ; x)$ corresponding to the finite interval $(a, b)$ with $\log p(x) /[(x-a)(b-x)]^{1 / 2}$ integrable $(L)$. The author derives the asymptotic expressions of $\phi_{n}(p ; z)$ for $n \rightarrow \infty$, also of $\phi_{n}{ }^{(i)}(p ; z)$ and of $\sum_{k=0}^{n} \phi_{k}{ }^{(i)}(p ; z) \phi_{k}^{(l)}\left(p ; z_{1}\right),(i, l=0,1, \cdots$ finite), the real points $z, z_{1}$ being arbitrarily taken outside of $(a, b)$. The method applied is very elementary. The first expression is derived without the use of difference 
or differential equations but by means of some previously established properties of algebraic continued fractions. The second expression is obtained from a difference equation of the first order. Some of these results have been presented to the French Academy (Comptes Rendus, vol. 183, (1926) p.697).

21. Professor E. L. Dodd: The convergence of general means of measurements, and the invariance of form of certain frequency functions.

With continuous increasing functions $f_{i}(u)$, let

$$
v=F(u)=\frac{1}{n} \sum_{1}^{n} f_{i}(u), u=G(v), M=G\left[\frac{1}{n} \sum_{1}^{n} f_{i}\left(m_{i}\right)\right],
$$

where $m_{\boldsymbol{i}}$ are measurements. Conditions are given for a probability greater than $1-\eta$ that $|M-a|<\epsilon$, where $a$ is the true value and $\epsilon$ and $\eta$ are preassigned, small at pleasure. A certain invariance of form of the associated frequency functions is shown. Moreover, if the probability that an error be negative is $1 / 2$, which does not require any symmetry in the frequency function, then the probability law for $M$ can be expressed in simple form by the use of the common probability integral.

\section{Dr. C. F. Roos: Generalized Lagrange problems in the • calculus of variations.}

In the theory of dynamical economics a problem arises which is a generalization of the Lagrange problem in the calculus of variations. It is desired to find in the space $u_{1}, u_{2}, u_{3}, x$ a curve $\Gamma$ which is a solution of a first order differential equation, which maximizes an integral $\pi_{1}$ when $u_{2}$ is kept fixed and which at the same time maximizes a second integral $\pi_{2}$ when $u_{1}$ is fixed. By employing the theory of Volterra integral equations, Eulerian necessary conditions are obtained without the use of multipliers. By allowing one end point to vary, conditions similar to the Weierstrass necessary condition are obtained. Sufficient conditions are then found by means of an analogue of the Hilbert integral. A similar treatment is given of the Lagrange problem with one integral but with several differential equations. It is shown that the analysis applies also to the problem in which the differential equations are replaced by functional equations.

\section{Dr. C. F. Roos: Dynamical economics.}

The hypotheses of Cournot, Walras and others concerning the equation of demand are modified so that the rate of change of price and production enter into the demand equation. Necessary conditions for a solution of the problems of competition, cooperation and monopoly are given for the case where the demand equation can be taken as a differential equation of the type $G\left(u_{1}, u_{1}{ }^{\prime}, \cdots, u_{n}, u_{n}{ }^{\prime}, p, p^{\prime}, t\right)=0$. In order to take account of the cumulative effects of price changes it is suggested that the differential equation be replaced by a functional equation. Some economic experiments which seem to support this theory are stated in brief. The mathematical 
problem of competition is then discussed from the view point of Walras and Pareto, using functional equations of demand.

\section{Professor G. A. Miller: Possible orders of two generators of the alternating and of the symmetric group.}

If $l>3$ represents a prime number which divides the order of the symmetric group of degree $n \neq 2 l-1$ then it is always possible to find two operators of orders 2 and $l$ respectively which generate this symmetric group and also two such operators which generate the alternating group of this degree. When $n=2 l-1$ it is possible to find two such generators of the alternating group of degree $n$ but it is impossible to find two such generators of the symmetric group of this degree. Every symmetric group whose order is divisible by 4 except the symmetric group of degree 6 can be generated by two operators of orders 2 and 4 respectively, and every alternating group which involves operators of order 4 can be generated by two such operators. Whenever an alternating group involves a substitution of order $l_{1}>3$ then it contains two substitutions of orders 2 and $l_{1}$ respectively which generate the entire group, and the theorems noted above include all the cases when the symmetric group cannot be thus generated. The paper will be offered for publication to the Transactions of this Society.

\section{Professor W. A. Manning: Simply transitive primitive} groups.

Dr. E. R. Bennett (American Journal of Mathematics, vol. 34 (1912)) gave a series of useful theorems on a restricted class of simply transitive primitive groups. The present paper obtains the conclusion contained in Corollary II to Theorem V of this paper from much weaker hypotheses, by proving the following theorem: Let $G_{1}$, the subgroup that leaves fixed one letter of the simply transitive primitive group $G$ of degree $n$ and order $g$, have a transitive constituent $M$ of degree $m$, in which the subgroup $M_{1}$ that fixes one letter is primitive. Let $M$ be "paired with itself" in $G_{1}$ and let the order of $M$ be less than $g / n$. Then $G_{1}$ contains an imprimitive constituent in which there is an invariant intransitive subgroup with $m$ transitive constituents of $m-1$ letters each, permuted according to the substitutions of $M$. This pairing of two transitive constituents of $G_{1}$ (or the pairing of a transitive constituent of $G_{1}$ with itself) was introduced by Burnside in his paper on Groups of odd order (Proceedings of the London Mathematical Society, vol. 33 (1900)). The author also extends and simplifies Theorems I to VI of Bennett's paper.

\section{Professor L. E. Dickson: Integers represented by} ternary quadratic forms.

One part of this paper has appeared in the January-February issue of this Bulletin. The other part will appear in the Annals of Mathematics. 
27. Professor L. E. Dickson: Quaternary quadratic forms representing all integers.

There is developed a method for finding all such quadratic forms, involving cross-products. The memoir will appear in the American Journal.

28. Professor L. E. Dickson: Extensions of Waring's theorem on sums of powers.

The part dealing with fourth powers will appear in an early issue of this Bulletin. The part concerning cubes will appear in the American Mathematical Monthly.

The form $a x^{3}+b y^{3}+\cdots$ is denoted by $(a, b, \cdots)$. From it is derived $(r, s, b, \cdots)$ by partition of $a$ into $r+s$. The latter form evidently represents every integer which can be represented by the first form. Call $a+b+\cdots$ the weight of the form. If a form of minimum weight 9 represents all positive integers, it is $f=(1,1,2,2,3)$ or one of the six forms derived from $f$ by partition. It is shown that $f$ represents all integers $<1400$. One of its partitions is $g=S+3 v^{3}$, where $S$ is a sum of six cubes; $g$ represents all integers $<40,000$. Many further forms are discussed, including all of weights 10 and 11 . In particular, $S+4 v^{3}$ represents all integers $<40,000$.

29. Mr. John Williamson: Conditions for associativity of division algebras corresponding to non-abelian groups.

It has been shown by L. E. Dickson (Transactions of this Society, April, 1926) that with every solvable group $G$ there is connected a system of division algebras. In this paper the conditions that the division algebras be associative are obtained when the group $G$ is generated by three independent generators $\Theta_{1}, \Theta_{p}$ and $\Theta_{q}$, where $\Theta_{p}$ transforms $\Theta_{1}$ into some power of $\Theta_{1}$, and $\Theta_{q}$ transforms $\Theta_{1}$ and $\Theta_{p}$ into some power of $\Theta_{1}$ and $\Theta_{p}$ respectively.

\section{Professor E. J. Moulton: A note on attraction.}

In this note the author criticizes derivations of the formulas for the attraction of a continuous body on a particle under the Newtonian Law of gravitation. Various writers, in an effort to improve the usual methods of mechanics, have used some formulation of Duhamel's Theorem. The author calls attention to an error introduced by some of these writers (W. F. Osgood, Annals of Mathematics, (2), vol. 4 (1903), p. 161; R. L. Moore, Annals of Mathematics, (2), vol. 13 (1912), p. 161; G. A. Bliss, Annals of Mathematics, (2), vol. 16 (1914), p. 45). They assume that the attraction of an element of the body is greater than the attraction of a particle of equal mass which is placed at the maximum distance from the given particle to a point of the element. This assumption is invalid.

\section{ARnold Dresden, Assistant Secretary.}

